\title{
Produção de materiais psicoeducativos a gestores da saúde para intervenção na pandemia da Covid-19
}

\author{
Production of materials to for health managers for \\ intervention in the Covid-19 pandemic
}

Daiane Zanqueta ${ }^{1}$, Letícia Accorsi², Maria Rita Zoéga Soares ${ }^{3}$, Silvia Regina de Souza ${ }^{4}$, Edmarcia Manfredin Vila ${ }^{5}$

\begin{abstract}
1. ORCID: https://orcid.org/0000-0001-9770-8352. Psicóloga. Mestranda em Análise do Comportamento pela Universidade Estadual de Londrina -- UEL. Londrina, - Paraná -, Brasil. E-mail: daianezanqueta22@gmail.com.

2. ORCID: https://orcid.org/0000-0001-9391-2989. Psicóloga. Mestranda em Análise do Comportamento pela Universidade Estadual de Londrina - UEL. Londrina -, Paraná -, Brasil). E-mail: leaccorsi14@gmail.com.

3. ORCID: https://orcid.org/0000-0002-6712-5209. Psicóloga. Doutora em Psicologia. Docente do Programa de Pós Graduação em Análise do Comportamento da Universidade Estadual de Londrina - UEL. Londrina -, Paraná -, Brasil. E-mail: ritazoega@hotmail.com.
\end{abstract}

4. ORCID: https://orcid.org/0000-0002-1496-8114. Psicóloga. Doutora em Psicologia Clínica. Docente do Departamento de Psicologia e Análise do Comportamento da Universidade Estadual de Londrina - UEL. Londrina -, Paraná -, Brasil. E-mail: ssouza.arrabal@gmail.com.

5. ORCID: https://orcid.org/0000-0002-0833-7455. Psicóloga. Doutora em Educação. Docente do Departamento de Psicologia e Análise do Comportamento da Universidade Estadual de Londrina - UEL. Londrina -, Paraná -, Brasil. E-mail: vila@uel.br.

CONTATO: Maria Rita Zoéga Soares | Rua João Wyclif, 185. Apto. 1303. Gleba Palhano. 86050450. Londrina - PR. Telefone: 43999175552 | E-mail: ritazoega@hotmail.com

RESUMO Intervenção psicológica é uma condição fundamental de saúde pública para superar a pandemia do Coronavirus Disease 2019. Profissionais da saúde e gestores, que estão na linha de frente no combate a essa doença, lidam diariamente com medo, ansiedade e estresse. Este relato de experiência descreve o processo de elaboração de um material psicoeducativo para gestores, a fim de orientá-los no manejo das variáveis ambientais que possam 
afetar a saúde mental dos trabalhadores sob sua responsabilidade. O material foi elaborado por docentes e discentes de Psicologia da Universidade Estadual de Londrina e colaboradores do projeto "Suporte Covid-19: atendimento aos profissionais e pacientes do Hospital Universitário". Os conteúdos foram elaborados com base em documentos específicos sobre como atuar em situação de estresse, desenvolvidos pela Fundação Oswaldo Cruz e Sociedade Brasileira de Psicologia. O material produzido foi disponibilizado on-line e por meio de cartazes fixados no hospital.

DESCRITORES: Covid-19. Gestores. Psicologia.

\begin{abstract}
Psychological intervention is a fundamental public health condition to overcome the Coronavirus Disease 2019 pandemic. Health professionals and managers, who are at the forefront in combating this disease, deal with fear, anxiety and stress on a daily basis. This experience report describes the process of preparing psychoeducational material for managers, in order to guide them in the management of environmental variables that may affect the mental health of the workers under their responsibility. The material was prepared by professors and students of Psychology at the State University of Londrina and collaborators in the project "Support Covid-19: care for professionals and patients at the University Hospital. The contents were prepared based on specific documents on how to act in a stressful situation, developed by the Oswaldo Cruz Foundation and the Brazilian Psychological Society. The material produced was made available online and through posters posted at the hospital.
\end{abstract}

DESCRIPTORS: Covid-19. Managers. Psychology.

\title{
INTRODUÇÃO
}

\footnotetext{
C oronavirus Disease 2019 (Covid-19) configura-se como uma situação de emergência e um dos fenômenos de maior impacto para a saúde pública nas últimas décadas ${ }^{1}$. A complexidade desta condição e o despreparo de diferentes setores da saúde para lidarem com o impacto da doença, têm favorecido problemas relacionados a à saúde mental de trabalhadores dessa área, indicando a necessidade de programas que visem ao desenvolvimento de repertórios comportamentais desses profissionais. Intervenções psicológicas têm sido citadas por alguns autores ${ }^{2}$ como necessárias para que esses profissionais possam lidar com o impacto da pandemia na sua vida.
} 
Contudo, o desenvolvimento de intervenções, em especial aquelas executadas remotamente, carecem de materiais (e.g., cartilhas, folders, entre outros) elaborados e avaliados a partir de uma teoria da aprendizagem. Quando se trata da Covid-19, é de suma importância a elaboração de recursos que orientem gestores a manejarem variáveis ambientais que possam afetar a saúde mental dos trabalhadores sob sua responsabilidade.

Desde janeiro de 2020, a Covid-19 foi considerada pela Organização Mundial da Saúde (OMS), uma Emergência de Saúde Pública de Interesse Internacional, devido ao surto da doença ocorrido na China³. A partir daí, o número de pesquisas em busca de respostas sobre a transmissão e disseminação da doença, riscos à saúde e meios de prevenção, aumentaram. Apesar do uso de máscaras e da higienização das mãos auxiliarem na diminuição da transmissão do vírus, o isolamento ainda é a melhor maneira de diminuir o contágio ${ }^{4}$, já que vacinas ainda estão sendo desenvolvidas e testadas. Nesse contexto, os profissionais da saúde estão mais suscetíveis a à contaminação pelo vírus em relação às demais pessoas.

Um estudo mostrou que a propagação do vírus em aerossol aumenta a distância de transmissão em até 4 metros dentro de UTIs em hospitais 5 . Além dos fatores de risco para a saúde física, também há fatores de risco para a saúde mental de profissionais que atuam na linha de frente ao combate à doença. Entre eles: alta exposição às pessoas infectadas pelo novo coronavírus, número de casos com necessidade de hospitalização e cuidados em unidades de terapia intensiva, escassez de suprimentos, ausência de intervenções terapêuticas eficazes e seguras (medicamentos e vacinas), longas horas de trabalho, óbito de pacientes, alta exigência para a tomada de decisões rápidas e eficazes, pouco controle da situação, medo de contrair a doença e infectar familiares etc. Além disso, há a preocupação com a possibilidade eminente de colapso do sistema de saúde, a imprevisibilidade acerca do tempo de duração da pandemia e dos seus desdobramentos sobre a populaçãó.

O profissional da saúde, como qualquer outro indivíduo, também tem de 
lidar com dificuldades no âmbito econômico, isolamento social e mudanças na rotina ${ }^{7}$. Todo esse cenário pode produzir e/ou agravar sintomas relacionados ao estresse, assim como, tristeza, confusão, raiva, ansiedade e depressão ${ }^{8-9}$. Ademais, há na literatura relatos de casos de suicídio, em alguns países, relacionados aos impactos psicológicos da Covid-1910.

Em razão dos impactos do referido contexto sobre a saúde mental de profissionais da área da saúde, intervenções psicológicas têm sido relatadas ${ }^{11-13}$, bem como descritos fatores importantes para o desenvolvimento de estratégias de intervenção em saúde mental em instituições. Segundo pesquisadores ${ }^{14}$, tais fatores estariam relacionados a à presença de equipes multidisciplinares (psiquiatras, psicólogos, assistentes sociais etc.), uso de dispositivos de comunicação clara (atualizados regularmente e seguros), desenvolvimento de serviços de aconselhamento/assessoria (propostas psicoeducativas com cartilhas e outros materiais informativos) e disponibilização de canais de comunicação (linhas telefônicas ou plataforma online) para esclarecimento de dúvidas. São recomendadas intervenções dinâmicas e pontuais, com o intuito de focar nas dificuldades da situação ou no seu manejo ${ }^{15}$.

Na tentativa de promover intervenções que contribuíssem para melhorar a saúde mental de médicos e enfermeiros, pesquisadores ${ }^{11}$ relataram intervenções psicológicas que envolveram: oferecer um lugar para que os profissionais pudessem descansar e se isolar temporariamente da família; gravar rotinas do hospital que poderiam ser compartilhadas pelos profissionais com suas famílias, aliviando as preocupações dos familiares; disponibilizar treinamento sobre como lidar com pacientes após confirmação da doença; apoiar a equipe para lidar com pacientes não cooperantes; desenvolver regras detalhadas sobre o uso e gerenciamento de equipamentos de proteção; realizar atividades de lazer; ensinar estratégias de relaxamento para reduzir o estresse e visitas regulares de psicólogos para identificar dificuldades de profissionais e fornecer apoio quando necessário. De acordo com os autores, as intervenções implementadas permitiram maior bem-estar dos profissionais. 
A necessidade de distanciamento social, devido a à possibilidade de contágio, tem levado os profissionais a implementarem estratégias de intervenções remotas, sugeridas por alguns pesquisadores ${ }^{12-13,15}$. O uso de aconselhamento remoto por escrito (terapia estruturada por cartas -- Structured Letter Therapy) foi descrito por Xiao 12. De acordo com o autor, o aconselhamento remoto por escrito pode combinar consulta, diagnóstico e tratamento. Apesar de se apresentar como uma possibilidade em época de quarentena, o aconselhamento remoto por escrito possui desvantagens que precisam ser melhoradas, como o fato de impossibilitar o manejo de crises psicológicas repentinas.

Ainda, pesquisadores chineses ${ }^{13}$ descreveram uma intervenção realizada com médicos, pacientes e outras pessoas afetadas, cujo objetivo foi auxiliar na superação de dificuldades psicológicas. A intervenção foi realizada por meio de serviços remotos (telefone e internet) para um grupo de pessoas de áreas afetadas e para equipes médicas da linha de frente que não foram infectadas de forma presencial. Psiquiatras e psicólogos realizaram avaliações dessas populações que eram então encaminhadas para atendimento, caso fosse necessário. Apesar de os autores não descreverem em detalhes os procedimentos empregados, relataram dificuldades com o atendimento remoto, como: ausência de histórico médico e de dados psicométricos, impossibilidade de visualizar a linguagem corporal, entre outros. Afirmaram que o uso desse tipo de intervenção deveria ser empregado apenas em situações emergenciais, como no caso da Covid-19.

Outros pesquisadores chinese ${ }^{15}$ também desenvolveram um modelo de intervenção em crises psicológicas que faz uso da tecnologia da Internetinternet. Esta alternativa integra médicos, psiquiatras, psicólogos e assistentes sociais nas plataformas da Internet internet para realizar intervenções psicológicas com pacientes, familiares e equipe médica. Esse modelo foi adaptado para se adequar aos diferentes estágios da pandemia (durante e após o surto). O reconhecimento dos diferentes estágios da pandemia e seus efeitos sobre os participantes foram importantes contribuições do trabalho. Contudo, devem ser 
considerados problemas referentes à utilização eficaz dos recursos/ferramentas da internet e a cooperação eficiente entre equipes médicas e psicólogos.

A despeito das dificuldades elencadas nos estudos supracitados, há consenso na literatura sobre a necessidade de se envidar esforços na busca de alternativas de intervenção psicológica direcionadas aos profissionais da área da saúde. Tal intervenção deveria minimizar os efeitos negativos da situação atual da pandemia sobre a saúde mental desses profissionais. A Psicologia pode contribuir auxiliando na promoção da saúde mental e na prevenção de implicações emocionais negativas para profissionais da área da saúde, oferecendo suporte e orientação sobre como manejar a situação, realizando-realizando -intervenções voltadas à orientação sobre sintomas psicológicos a esses profissionais ${ }^{15}$ e abordando estratégias de enfrentamento, autocuidado e gerenciamento de estresse ${ }^{16}$. As estratégias de intervenção devem contribuir principalmente para o fortalecimento da rede de apoio ${ }^{11}$.

Outros aspectos importantes a serem abordados sobre os profissionais da saúde é a qualidade do sono e o apoio social, prejudicados nesse contexto, e que influenciam no aumento do estresse e da ansiedade ${ }^{12}$. $O$ apoio de outros profissionais da saúde, como gestores, colegas de equipe e da família contribuem para melhorar a autoeficácia e reduzir os níveis de ansiedade e estresse enfrentados por esses profissionais ${ }^{12}$. Embora muitas das intervenções conduzidas nessa área tenham trabalhado diretamente com médicos e enfermeiros que atuam na linha de frente, orientações podem ser fornecidas a gestores.

Gestores de saúde tem têm funções importantes no ambiente hospitalar que vão além da liderança e da gestão dos vários setores organizacionais da saúde ${ }^{17}$. Cabe a eles: utilizar estratégias para que os profissionais compreendam os objetivos da organização; auxiliar no desenvolvimento da motivação da equipe; utilizar comunicação aberta e contínua com todos; promover autonomia no processo de decisão da equipe; auxiliar no desenvolvimento de vínculos entre os profissionais; fomentar a criatividade e mudança; e; liderar todos os profissionais envolvidos no ambiente de saúde, como médicos, enfermeiros, 
fisioterapeutas, psicólogos, profissionais da administração, da limpeza, da segurança, da manutenção, entre outros ${ }^{17}$.

Os gestores têm importantes ferramentas administrativas e de organização de pessoal que podem contribuir para o manejo e o bem-estar, tanto de sua equipe quanto deles mesmos. Eles podem organizar condições para prevenir e proteger o grupo contra o estresse crônico e contribuir para que todos exerçam, da melhor forma possível, suas funções durante a crise da Covid-19. Contudo, a falta de experiência dos gestores para lidarem com situações de pandemia, sugere a necessidade de treinamento que os auxilie no desenvolvimento de repertório para soluções rápidas e eficientes. Gestores que atuam em instituições ou com profissionais que estão na linha de frente ao combate à Covid-19, podem se sentir vulneráveis para lidarem com as situações estressoras advindas das demandas neste período. De acordo com pesquisadores britânicos ${ }^{18}$, os gestores de saúde em cargos de supervisão devem reconhecer o desafio enfrentado pela sua equipe e minimizar os riscos psicológicos inerentes às atividades que desenvolvem como, por exemplo, lidar com dilemas difíceis. Apesar da importância dos gestores em tempos de Covid-19, são escassos os trabalhos que visam a orientá-los sobre como contribuir para a saúde psicológica dos profissionais sob sua responsabilidade e os materiais produzidos que cumpram essa função.

O desenvolvimento de materiais como cartilhas, folders e manuais têm sido usados para capacitar e/ou orientar profissionais que atuam em diferentes contextos. No contexto da saúde, guias, cartilhas ou manuais, buscam informar sobre um assunto específico e proporcionar condições para desenvolver repertório para o enfrentamento da situação ${ }^{19}$. Esses materiais devem ser elaborados de maneira a auxiliar a compreensão do público-alvo, com estratégias didáticas e imagens com linguagem clara e acessível para favorecer a aprendizagem de habilidades ${ }^{19}$. O uso de materiais escritos pode auxiliar na compreensão das informações, comunicação, esclarecimento de dúvidas e auxílio nas tomadas de decisões ${ }^{20}$. 
No contexto de saúde pública, cartilhas têm a função de levar informações e fornecer instruções sobre o que fazer diante de uma determinada situação. Manuais ou cartilhas podem auxiliar na adesão a proposta, generalização dos conteúdos e avaliação de habilidades ${ }^{22}$. Esses materiais elaborados na área da saúde possibilitam a prevenção e promoção da saúde ${ }^{19,21}$, além de serem uma alternativa ao trabalho com intervenções remotas.

Em razão da importância da atuação dos gestores para a manutenção da saúde física e mental dos profissionais da saúde sob sua responsabilidade, da recomendação para que neste período a intervenção psicológica ocorra por meio de plataformas remotas ${ }^{11}$ e do potencial de cartilhas para fornecer orientações, o presente artigo tem como objetivo, descrever o processo de elaboração de um material psicoeducativo para gestores, a fim de orientar o manejo das variáveis ambientais que possam afetar a saúde mental dos trabalhadores sob sua responsabilidade. A cartilha foi produzida como uma das atividades do projeto "Suporte Covid-19: atendimento aos profissionais e pacientes do Hospital Universitário da Universidade Estadual de Londrina (UEL)", realizado por docentes e discentes da UEL e colaboradores.

\section{MÉTODO}

A proposta da cartilha surgiu por meio de um projeto de atendimento psicológico e tem o intuito de fornecer apoio psicológico aos profissionais da saúde que trabalham na linha de frente da Covid-19. O projeto intitulado "Suporte Covid-19: atendimento aos profissionais e pacientes do Hospital Universitário-UEL" " é composto por oito frentes, responsáveis por diferentes serviços, como a capacitação de Psicólogospsicólogos, a produção de materiais, o atendimento ao público, entre outros.

Um dos materiais desenvolvidos pela frente de Processos Educativos foi a cartilha para gestores. A cartilha foi elaborada em razão da demanda dos gestores relacionada ao estresse, a à alta carga de trabalho e a à falta de experi- 
ência da equipe em lidar com situações de pandemia. Tais fatores estariam prejudicando a saúde física e mental dos profissionais que atuavam na linha de frente do Hospital Universitário e UBSs, alvos de atendimento do projeto.

Para a elaboração da cartilha foram consultados materiais referentes a à Covid-19 e que apresentavam e discutiam intervenções nessa área com diferentes públicos. Apesar dos esforços da comunidade científica, a quantidade de materiais publicados sobre o tema ainda é reduzida, se comparada a outras áreas de investigação, e muitos dos trabalhos publicados ainda não apresentam dados para respaldar a efetividade das intervenções propostas. A análise dos materiais encontrados indicou escassez de trabalhos que discutiam intervenções ou desenvolvimento de materiais cuja finalidade era orientar gestores a manejarem variáveis ambientais que afetariam a saúde mental de trabalhadores sob sua responsabilidade.

Assim, os temas abordados na cartilha seguiram as orientações de materiais desenvolvidos pela Fundação Oswaldo Cruz (Fiocruz) ${ }^{23}$ e pela Sociedade Brasileira de Psicologia (SBP) ${ }^{24}$. O material da Fiocruz apresenta recomendações aos gestores para ações relacionadas à Saúde Mental e Ações Psicossociais na Pandemia (SMAPS). A fim de oferecer orientações técnicas a profissionais da psicologia, a SBP, por meio do "Grupo de Trabalho (GT) de enfrentamento da Pandemia SBP Covid-19", reuniu informações técnicas atualizadas para contribuir com a prática profissional da Psicologia. Para a elaboração da cartilha foi usado o material da SBP ${ }^{24}$, que oferecia aos psicólogos, alternativas para o manejo do estresse em profissionais da área da saúde em geral.

Após a leitura desses materiais, identificaram-se temas que poderiam ser usados para a orientação de gestores. Foram selecionados aqueles que poderiam auxiliar no manejo de variáveis ambientais que afetariam a saúde mental dos trabalhadores sob sua responsabilidade. Os temas gerais foram discutidos entre profissionais psicólogos, participantes do referido projeto. Os temas selecionados foram elencados em itens. Em seguida, cada um dos itens foi avaliado e decomposto em orientações mais específicas. Por fim, os profissionais 
avaliaram a linguagem quanto a à clareza e ortografia e, encaminharam para profissionais da área do design para a execução do layout.

\section{RESULTADOS}

A partir da literatura foram selecionados seis objetivos gerais, ou comportamentos-objetivo, que deveriam ser apresentados pelos gestores para minimizar riscos psicológicos inerentes ao lidar com situações difíceis, como é o caso da pandemia. Comportamentos-objetivo são comportamentos que se espera que o aprendiz, no caso o gestor, seja capaz de apresentar com o uso das informações da cartilha. O Quadro 1 descreve tais comportamentos.

Quadro 1. Lista dos comportamentos-objetivo gerais e intermediários selecionados para a cartilha.

\section{COMPORTAMENTOS-OBJETIVO GERAIS E INTERMEDIÁRIOS SELECIONADOS}

1.Definir claramente o papel e a responsabilidade de cada profissional.

- Realizar reuniões pontuais, no início e no final de cada dia de trabalho, para definir as tarefas de cada equipe e de cada pessoa envolvida;

- Elaborar atas dessas reuniões, que permitam que os profissionais possam recorrer a elas em casos de dúvidas;

- Não responsabilizar profissionais que estejam passando por dificuldades ou que precisem se ausentar em algum momento.

\section{Incentivar a comunicação e a atualização dos profissionais sob sua responsabilidade.}

- Realizar reuniões em que todos os profissionais possam se posicionar, fornecer feedbacks sobre a atuação de si mesmo e dos outros, e apresentar problemas enfrentados na semana ou no dia;

- Ouvir o relato dos colegas sobre o impacto dos casos atendidos;

- Ouvir atentamente e sem julgar os profissionais de saúde que estiverem 
passando por sofrimento;

- Elogiar o empenho e esforço de todos os profissionais.

\section{Incentivar o suporte social entre os colegas de trabalho.}

- Auxiliar os funcionários mais impactados pela situação ou que tenham familiares afetados pela enfermidade;

- Solicitar aos profissionais que prestem auxílio aos colegas que estiverem emocionalmente mais vulneráveis;

- Fornecer feedback claro e frequente à equipe, evitar broncas, tratamentos rudes e priorizar instruções e elogios;

- Sinalizar quando a atitude de um profissional ofender o outro;

- Conversar em particular quando a atitude de um profissional ofender a outrem, colega ou qualquer outra pessoa;

- Ser cortês com outros profissionais do hospital (usar locuções como: "por favor", "com licença”, "desculpe" etc.).

\section{Incentivar as chefias a ouvirem as sugestões e feedbacks dos profis- sionais.}

- Acolher as sugestões dos profissionais e das chefias, quando for pertinente;

- Evidenciar as habilidades e competências de cada profissional, diante das chefias;

- Evidenciar as contribuições dos profissionais e das chefias para o manejo de situações-problema do cotidiano.

\section{Contribuir para o manejo do estresse.}

- Monitorar o bem-estar da equipe;

- Fazer rodízio dos profissionais entre ambientes de alto e baixo nível de estresse;

- Fazer rodízio dos profissionais com alto e baixo nível de experiência;

- Sinalizar a necessidade de os profissionais realizarem atividade física, descansarem e se alimentarem de modo saudável; 
- Salientar a importância de pausas breves para descanso e recuperação durante a jornada de trabalho;

- Garantir que a equipe tenha o descanso e a recuperação necessária;

- Esclarecer aos profissionais que o estresse é esperado em situações de crise;

- Informar os profissionais de que se sentir estressado não significa fraqueza nem incapacidade para realizar o trabalho;

- Apontar o risco de utilizar estratégias negativas de manejo do estresse (abuso de substâncias lícitas e ilícitas, como, por exemplo, o álcool etc.);

- Disponibilizar e indicar atendimento de saúde mental para os profissionais.

\section{Executar ações de autocuidado.}

- Alimentar-se da melhor maneira possível;

- Estabelecer, para si próprio, breves períodos de pausa durante a jornada de trabalho;

- Restringir as preocupações de trabalho ao ambiente do hospital sempre que possível;

- Aproveitar os momentos do contraturno de trabalho para conversar sobre diferentes assuntos com familiares e amigos e/ou realizar outras atividades que sejam prazerosas, como: escutar música, ver filmes, ler, cozinhar;

- Conversar com colegas de trabalho sobre seu sofrimento em relação ao momento de crise;

- Lembrar que seu sofrimento não é sinal de fraqueza nem incapacidade.

Fonte: Elaborado pelas autoras (2020).

Os Objetivos objetivos de 1 a 5 foram retirados do material da $\mathrm{SBP}^{24}$ e o Objetivo objetivo 6 foi incluído pois os gestores também estariam submetidos a condições de trabalho estressantes e, portanto, também deveriam ser alvo da intervenção. Considerando a abrangência dos comportamentos-objetivo gerais, cada um deles foi decomposto em comportamentos intermediários a fim de melhor operacionalizá-los. O Comportamento-objetivo 1 (definir claramente 
o papel e a responsabilidade de cada profissional) implica em instruções ao gestor sobre como auxiliar os profissionais sob sua responsabilidade a organizar as atividades ao longo da rotina de trabalho, definindo as metas e como os profissionais poderiam alcançá-las. Definir claramente as metas e objetivos da equipe, além do papel de cada um para que essa meta possa ser alcançada, contribui para que todos trabalhem em uma mesma direção, produzindo meIhores resultados.

O Comportamento-objetivo 2 (incentivar a boa comunicação e a atualização sobre informações relevantes) envolve a apresentação de instruções sobre a importância de o gestor proporcionar um local onde as informações sejam veiculadas e onde os profissionais possam expressar suas opiniões sobre os problemas enfrentados no dia a dia. Espera-se um contexto de acolhimento de relatos verbais e valorização do trabalho realizado. A boa comunicação entre gestores e demais profissionais pode contribuir para a melhor integração entre eles, auxiliando na realização das atividades diárias e na compreensão das informações necessárias à execução do trabalho. O profissional deve se sentir à vontade para expressar suas dificuldades e ter seus sentimentos validados, transformando o local de trabalho em um ambiente acolhedor. Também se enfatiza a importância do elogio no sentido de incentivo e reconhecimento pela emissão do comportamento desejado.

O Comportamento-objetivo 3 (incentivar o suporte social entre os colegas de trabalho) fornece dicas sobre como os gestores podem oferecer suporte social adequado aos profissionais que compõem sua equipe, bem como contribuir para a formação de redes de apoio social e para um ambiente agradável de trabalho. As relações interpessoais têm um importante papel na forma como reagimos às adversidades, influenciando na qualidade de vida e saúde mental.

O Comportamento-objetivo 4 (incentivar as chefias a ouvirem as sugestões e feedbacks dos profissionais) apresenta aos gestores a importância da comunicação entre ele e a equipe, principalmente, a importância de ouvir a todos. Saber ouvir e aceitar as contribuições dos membros da equipe pode melhorar 
o engajamento nas tarefas propostas e possibilitar tomadas de decisões mais convenientes, considerando que o profissional que vivencia a situação é o que mais tem conhecimento sobre ela. O papel do gestor nesse diálogo entre chefia e profissionais é fundamental para que se possa avaliar as estratégias empregadas e elaborar alternativas de intervenção adaptadas às demandas do contexto.

O Comportamento-objetivo 5 (contribuir para o manejo do estresse) está relacionado ao gestor ser capaz de manejar variáveis ambientais relacionadas ao estresse. Aborda a importância da interação entre o gestor e sua equipe e traz orientações sobre como ele pode contribuir para a prevenção do estresse. O estresse contínuo pode trazer consequências prejudiciais à saúde mental tanto do gestor quanto dos profissionais de sua equipe. Nesta Nessa situação, é frequente o relato de distúrbios de sono e sintomas relacionados a ansiedade, depressão, maior propensão ao consumo de álcool e uso de substâncias psicoativas, o que contribui para o prejuízo da qualidade de vida.

Por fim, o Comportamento-objetivo 6 (tomar ações de autocuidado) tem por finalidade mostrar que as ações do gestor podem colaborar para a diminuição dos riscos psicológicos dos membros de sua equipe, incluindo ele mesmo. É fundamental que adotem estratégias de cuidado de si mesmos e que saibam manejar situações relacionadas ao estresse. As estratégias devem considerar o cuidado com aspectos físicos e mentais, na tentativa de auxiliá-los a identificar situações de sobrecarga de trabalho e excesso de preocupações. No contexto da pandemia, os gestores estão mais expostos a cargas de trabalho elevadas e com pouco tempo para o lazer. Contudo, para melhorar o bem bem-estar e a qualidade do trabalho neste período, é fundamental que saibam organizar a própria rotina e considerar a importância de momentos de descanso, relaxamento e lazer.

O material foi intitulado "Gestor da Saúde: como manejar situações de estresse em sua equipe". Apresenta uma introdução, na qual abordam-se aspectos referentes ao contexto vulnerável da pandemia nas redes de saúde pública 
e suas consequências para a saúde física e mental. Disponibiliza informações relevantes para serem trabalhadas no contexto da saúde pública, além da importância do papel do gestor da saúde na prevenção do estresse. Após a introdução, são apresentados os comportamentos-objetivo gerais e intermediários. Ressalta-se que na cartilha optou-se pelo uso dos verbos na forma imperativa (e.g., em vez de "incentivar" usou-se "incentive"), como condição motivadora para a leitura e a execução da ação. Por fim, foi incluída uma pequena conclusão para alertar o gestor quanto a à necessidade de pedir ajuda sempre que necessitar. O contato e o e-mail do projeto Suporte Psicológico Covid-19 também foram incluídos, em caso de necessidade.

Em razão da sobrecarga de trabalho desses profissionais e da falta de tempo para leitura, optou-se em por elaborar uma cartilha resumida e com orientações mais diretas sobre como proceder. O material contém 4 páginas e, para torná-lo atrativo, uma profissional do design executou o layout. A Figura 1 apresenta algumas páginas do material elaborado.

\section{DISCUSSÃO}

A pandemia de Covid-19 trouxe consigo condições de trabalho ainda mais estressantes para os profissionais de saúde, em especial, aqueles que estão na linha de frente no combate ao novo coronavírus. A vulnerabilidade relacionada ao alto índice de exposição e contaminação -- pelo contato direto com pacientes sintomáticos e diagnosticados com a infecção pelo vírus -- é considerada o principal fator de risco na atuação em saúde no momento. Além da maior probabilidade de contaminação, constatou-se que esses profissionais estavam lidando com situações favorecedoras ao surgimento de problemas relacionados à saúde mental, tais como: carga de trabalho excessiva, disponibilidade inadequada de equipamentos de proteção individuais (EPIs), isolamento de familiares, frustração, insegurança no trabalho, entre outros. Tais condições, quando suportadas por períodos longos, podem gerar consequências psicológicas diretamente relacionadas ao desenvolvimento de sintomas 
Figura 1. Páginas da cartilha para os gestores.
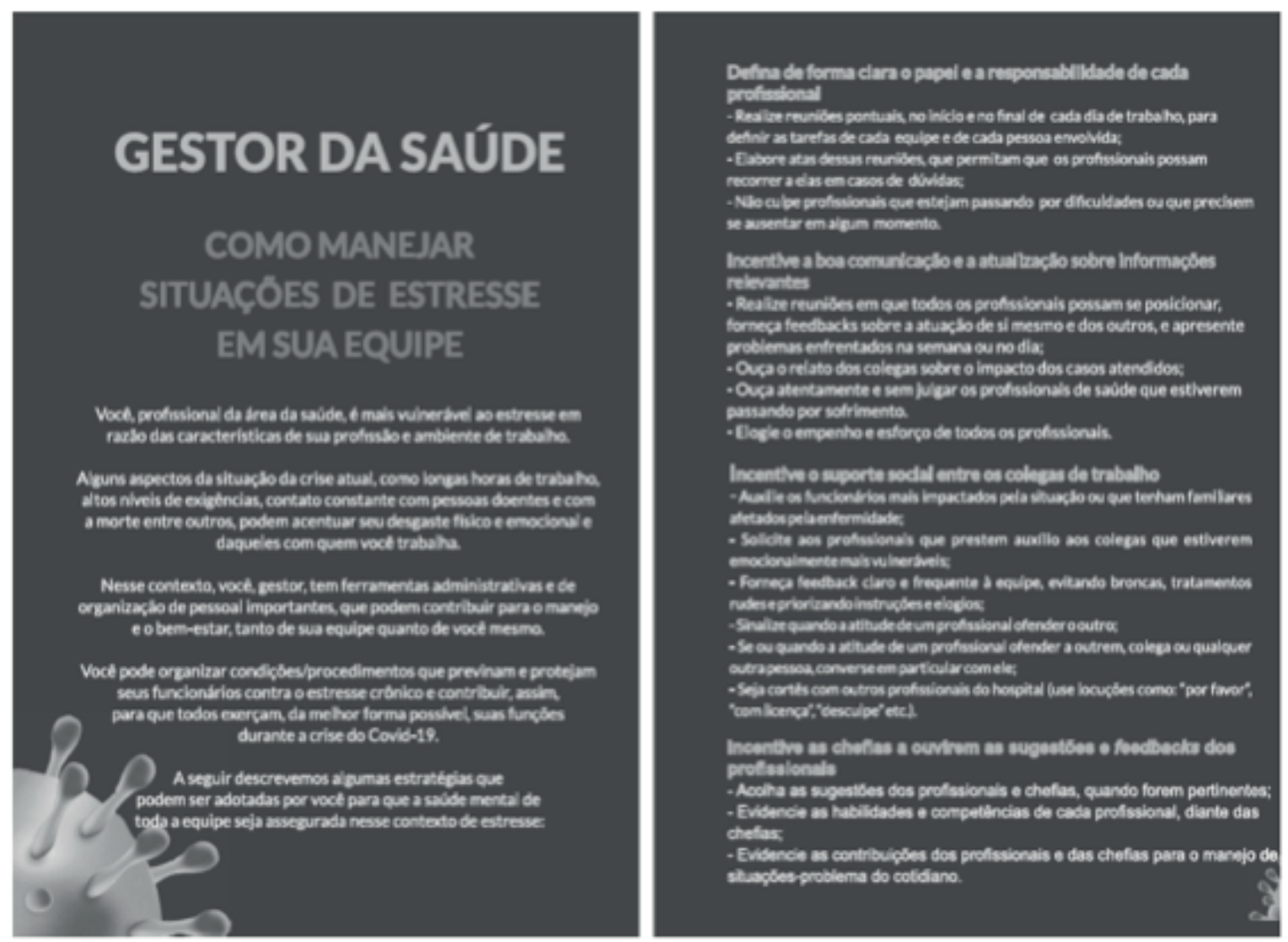

Fonte: Elaborado pelas autoras (2020).

de ansiedade, estresse, depressão, insônia, abuso de substâncias, entre outros ${ }^{8-9}$.

Gestores de serviços de saúde podem executar ações que promovam a proteção da saúde física e psicológica de profissionais que trabalham diretamente na linha de frente, atendendo pacientes infectados pelo Covid-19. Os gestores têm papel de liderança, realizando planejamentos, organizando e acompanhando atividades, mapeando prioridades, lidando com imprevisibilidades, tentando reduzir sobrecarga de trabalho, integrando equipes, monitorando metas, mediando conflitos, atuando em nível estratégico, organizacional e técnico. Tais ações representam um grande desafio, principalmente quando somadas à formação insuficiente, escassez de recursos financeiros e à realidade da prestação de serviços públicos em saúde no Brasil $25-26$. 
No que se refere à formação, o uso de materiais psicoeducativos escritos demonstrou ser uma alternativa viável para auxiliar gestores nesta situação. Esses materiais propiciaram a orientação de profissionais atuantes nos cargos de gestão, buscando proporcionar melhor qualidade de vida, garantindo condições que promovam a saúde mental e ofereçam melhor assistência em saúde coletiva ${ }^{27}$.

Materiais psicoeducativos impressos (e.g., folhetos, folders, manuais e cartilhas) são amplamente utilizados na área da saúde devido aos seus benefícios, como: baixo custo de produção por unidade de material; assimilação da linguagem de acordo com o ritmo de aprendizagem; possibilidade de leitura múltiplas; liberdade de escolha do profissional em relação ao tempo e local mais apropriado para seu uso e, ainda, possibilidade de implementação enquanto material complementar a outros tipos de intervenção ${ }^{20}$. Entretanto, o emprego desse tipo de material também apresenta limitações, como ter que ser elaborado de forma impessoal e não considerar as características individuais de cada leitor (e.g., repertório de habilidades). Além disso, não é possível avaliar o impacto do material sobre os gestores e profissionais, em decorrência da amplitude de sua disseminação.

Contudo, as vantagens desse tipo de tecnologia psicoeducacional para capacitação de profissionais excedem as limitações descritas, principalmente quando os demais formatos de intervenção tornaram-se impraticáveis. A elaboração adequada de materiais pode facilitar a descrição do problema, favorecer a compreensão e promover o desenvolvimento de estratégias profissionais do psicólogo, atuando enquanto educador em assuntos específicos ${ }^{28}$. A praticidade da aplicação do material psicoeducativo escrito representa uma possibilidade de intervenção coerente com a urgência da demanda apresentada por esses profissionais e permite o acesso rápido às informações sobre a atuação em equipes de trabalho.

Estudos corroboram a premissa de que o uso de manuais e cartilhas pode ser uma ferramenta de capacitação de diversas populações da área da saúde, apresentando estratégias favorecedoras à emissão de comportamentos adaptativos ${ }^{29-30}$ para pacientes com doenças crônicas (e.g., câncer, dermatite atópica e diabetes) ${ }^{29}$, cuidadores ${ }^{19}$ e profissionais de saúde ${ }^{30}$. 
As pesquisas supracitadas, em sua maioria, atestam a importância de atentar-se ao processo de construção de materiais psicoeducativos para alcançar critérios como legibilidade, compreensão, eficácia do material e engajamento. Assim, etapas como identificação de público-alvo para adequação de linguagem e conteúdo; identificação de canais de divulgação apropriados; desenvolvimento do material psicoeducativo por equipe multidisciplinar (e.g., psicólogos, designers, profissionais da saúde); e validação e avaliação do material produzido pelo público-alvo são indispensáveis para produzir materiais culturalmente relevantes ${ }^{20,28}$.

Seria importante que gestores e profissionais pudessem ter a oportunidade de fornecer feedback sobre o material e optar pela melhor alternativa para a divulgação, no sentido de contribuírem para a adequação e otimização do consumo do conteúdo. Mas, em consequência da urgente demanda apresentada pelos gestores para a produção do material, não foi possível realizar a etapa de validação social. Considera-se tal aspecto especialmente importante para avaliação da linguagem, quantidade de informação, layout e ilustrações utilizadas. Além disso, em função das demandas do hospital, optou-se pela disponibilização do material por meio de folder fixados nas áreas de circulação do hospital/UBSs e no formato on-line, com o objetivo de aumentar a acessibilidade dos profissionais de saúde. A implementação imediata dessa medida configura-se como uma ferramenta contingencial em um momento que exige criatividade para intervir.

\section{CONSIDERAÇÕES FINAIS}

A utilização de materiais educativos pode ser amplamente difundida na área da saúde, como alternativa para a formação e o engajamento de gestores que se preocupam com a saúde mental e qualidade de vida de sua equipe profissional. Levando em consideração as dificuldades vivenciadas por profissionais de saúde para atuar diante do contexto propiciado pela pandemia, o presente artigo buscou apresentar uma cartilha de orientação para gestores de saúde sobre como manejar situações relacionadas ao estresse e sofrimento em sua equipe de trabalho. 
Nesta situação de pandemia, a elaboração de materiais psicoeducativos deve incluir a participação de profissionais de diferentes áreas, que estão atuando na situação, além de seguir um roteiro que cumpre distintas etapas, como a busca na literatura, levantamento de comportamentos-objetivo a serem alcançados e validação com participação do público-alvo. Tais passos buscam assegurar que o material atenda as demandas advindas do contexto e se adeque às características dos profissionais envolvidos.

A cartilha elaborada foi direcionada aos gestores da saúde, por serem os responsáveis pela orientação e auxílio às práticas realizadas em ambientes de saúde pública. Além de possuírem acesso direto aos profissionais da saúde e poderem contribuir com a capacitação e orientação de um número maior de pessoas, muitas vezes não contemplados em intervenções psicológicas remotas.

A conjuntura atual da atuação em saúde reitera a importância de que os gestores de saúde, que lideram equipes, estejam habilitados a criar condições que proporcionem fatores de prevenção em saúde mental, além de apresentarem repertório comportamental sobre como realizar o manejo da situação. A intervenção psicológica deve ser direcionada ao desenvolvimento de habilidades de profissionais na área da saúde para lidarem com as dificuldades em momentos de crise.

\section{REFERÊNCIAS}

\footnotetext{
1. Faro A, Bahiano MA, Nakano TC, Reis C, Silva BFP, Vitti LS. COVID-19 e saúde mental: a emergência do cuidado. Estud Psicol (Campinas) [Internet]. Campinas: Estud. Psicol.; 2020. [acesso citado em 2020 jun Jun 30]; 37: e200074. Disponível em: https:// doi.org/10.1590/1982-0275202037e200074.

2. Schmidt B, Crepaldi MA, Bolze SDA, Neiva-Silva L, Demenech LM. Saúde mental e intervenções psicológicas diante da pandemia do novo coronavírus (COVID-19). Estud Psicol. (Campinas) [Internet]. Campinas: Estud. Psicol.; 2020. [acesso citado em 2020 jun Jun 30]; 37: e200063. Disponível em: https://doi.org/10.1590/1982-0275202037e200063.

3. Sohrabi C, Alsafi Z, O'Neill N, Khan M, Kerwan A, Al-jabir A et al.World Health Organization declares global emergency: A review of the 2019 novel coronavirus (COVID-19). Int J Surg (London, England) [Internet]. Londres: Int. J Surg.; 2020. [acesso citado em 2020 jul Jul 10]; 76: 71-76. Disponível em: https://doi.org/10.1016/j.jisu.2020.02.034.

4. Cascella M, Rajnik M, Cuomo A, Dulebohn SC, Di Napoli R. Features, Evaluation and Treatment Coronavirus (COVID-19). StatPearls [Internet]. StatPearls; 2020. [acesso citado em 2020 jul Jul 04]. Disponível em: https://www.ncbi.nlm.nih.gov/books/ NBK554776/.

5. Guo ZD, Wang ZY, Zhang SF, Li X, Li L, Li C et al. Aerosol and Surface Distribution of Severe Acute Respiratory Syndrome
} 
Coronavirus 2 in Hospital Wards, Wuhan, China. Emerg Infect Dis [Internet]. Emerg Infect Dis; 2020 [acesso citado em 2020 jul Jul 22]; 26(7): 1583-1591. Disponível em: https://doi.org/10.3201/eid2607.200885.

6. Zandifar A, Badrfam R. Iranian mental health during the COVID-19 epidemic. Asian journal of psychiatry [Internet]. Asian journal of psychiatry; 2020 [acesso citado em 2020 jul Jul 22]; 51: 101990. Disponível em: https://doi.org/10.1016/j.ajp.2020.101990.

7. Desclaux A, Badji D, Ndione AG, Sow K. Accepted monitoring or endured quarantine? Ebola contacts" perceptions in Senegal. Soc Sci Med [Internet]. Soc Sci Med; 2017. [acesso citado em 2020 jun Jun 30]; 178:38-45. Disponível em: https://doi. org/10.1016/j.socscimed.2017.02.009.

8. Brooks SK, Webster RK, Smith LE, Woodland L, Wessely S, Greenberg N et al. The psychological impact of quarantine and how to reduce it: rapid review of the evidence. Lancet [Internet]. Lancet; 2020. [acesso citado em 2020 jul Jul 22]; 395(10227): 912-920. Disponível em: http://dx.doi.org/10.1016/S0140-6736(20)30460-8.

9. Wang C, Pan R, Wan X, Tan Y, Xu L, Ho CS et al. Immediate psychological responses and associated factors during the initial stage of the 2019 coronavirus disease (COVID-19) epidemic among the general population in china. Int J Environ Res Public Health [Internet]. Int J Environ Res Public Health; 2020. [acesso citado em 2020 jul Jul 29]; 17(5): 1729. Disponível em: http:// dx.doi.org/10.3390/ijerph17051729.

10. Goyal K, Chauhan P, Chhikara K, Gupta P, Singh MP. Fear of COVID 2019: first suicidal case in India [Internet]. Asian Journal of Psychiatry [Internet].; 2020. [acesso citado em 2020 jul Jul 29]; 49(101989). Disponível em: http://dx.doi.org/10.1016/j. ajp.2020.101989.

11. Chen Q, Liang M, Li Y, Guo J, Fei D, Wang L et al. Mental health care for medical staff in China during the COVID-19 outbreak [Internet].. Lancet; [Internet]. 2020. [acesso citado em 2020 jul Jul 04]; 7(4): 15-16. Disponível em: http://dx.doi.org/10.1016/ S2215-0366(20)30078-X.

12. Xiao C. A novel approach of consultation on 2019 novel coronavirus (COVID-19)-related psychological and mental problems: structured letter therapy [Internet]. Psychiatry Investig [Internet]. ; 2020. [acesso citado em 2020 jun Jun 30]; 17(2): 175-176. Disponível em: http://dx.doi.org/10.30773/pi.2020.0047.

13. Jiang X, Deng L, Zhu Y, Ji H, Tao L, Liu L et al. Psychological crisis intervention during the outbreak period of new coronavirus pneumonia from experience in Shanghai [Internet]. Psychiatry Res; [Internet]. 2020. [acesso citado em 2020 jul Jul 23]; 286: 112903. Disponível em: http://dx.doi.org/10.1016/j.psychres.2020.112903.

14. Xiang YT, Yang Y, Li W, Zhang L, Zhang Q, Cheung T et al. Timely mental health care for the 2019 novel coronavirus outbreak is urgently [Internet].. Lancet [Internet]. ; 2020. [acesso citado em 2020 jul Jul 22]; 7(3): 228-229. Disponível em: https://doi. org/10/1016/S2215-0366(20)30046-8.

15. Zhang C, Yang L, Liu S, Ma S, Wang Y, Cai Z et al. Survey of insomnia and related social psychological factors among medical staff involved in the 2019 novel coronavirus disease outbreak [Internet]. Front Psychiatry; [Internet]. 2020(a). [acesso citado em 2020 jul Jul 22]; 11(306): 1-9. Disponível em: http://dx.doi.org/10.3389/fpsyt.2020.00306.

16. Taylor S. The psychology of pandemics: preparing for the next global outbreak of infectious disease. Newcastle upon Tyne: Cambridge Scholars Publishing; 2019.

17. Chanes M. Os desafios na formação de gestores líderes em saúde [Internet]. São Paulo: O Mundo da Saúde [Internet].; 2006. [acesso citado em 2020 jun Jun 30]; 30(2): 326-331. Disponível em: http://www.saocamilo-sp.br/pdf/mundo_saude/35/ desafios_formacao.pdf.

18. Greenberg N, Docherty M, Gnanapragasam S, Wessely S. Managing mental health challenges faced by healthcare workers during covid-19 pandemic [Internet]. BM] [Internet]. ; 2020. [acesso citado em 2020 jul Jul 29]; 368: m1211. Disponível em: https://doi.org/10.1136/bmj.m1211.

19. Martins FCM, Soares MRZ. (2019). Guia de Orientação: Uma estratégia de Intervenção para cuidadores de Pacientes Oncológicos. In: Gioia PS, Azoubel MS, organizadores. Estudos em Análise do Comportamento e Saúde. Curitiba: CRV; 2019. p.125-139.

20. Moreira MF, Nóbrega MML, Silva, MIT. Comunicação escrita: contribuição para a elaboração de material educativo em saúde. Rev Bras Enferm [Internet]. Rev Bras Enferm; 2003. [acesso citado em 2020 jun Jun 30]; 56(2): 184-8. Disponível em: https://doi.org/10.1590/S0034-71672003000200015.

21. Rosot N, Rocha MM, Fornazari SA. Elaboração de um material sobre habilidades sociais educativas para pais de crianças com TDAH. In: Costa CE, Souza SR, Haydu VB, organizadores. Psicologia: Avaliação e Intervenção Analítico comportamental. Londrina: Eduel; 2020. p.103-130.

22. Del Prette ZAP, Del Prette A. Enfoques e modelos do treinamento de habilidades sociais. In: Del Prette ZAP, Del Prette A, organizadores. Habilidades Sociais: intervenções efetivas em grupo. São Paulo: Casa do Psicólogo; 2011. p. 19-56. 
23. Ministério da Saúde. Fiocruz [Internet]. Brasil: Ministério da Saúde; 2020. [citado em 2020 jun Jun 30]. Disponível em: https://www.saude.gov.br/component/tags/tag/fiocruz.

24. Miyazaki MCOS, Soares MRZ. Estresse em profissionais da saúde que atendem pacientes com COVID-19. Sociedade Brasileira de Psicologia [Internet]. Brasil: SBP; 2020. [citado em 2020 jun Jun 30]. Disponível em: https://www.sbponline.org. br/2020/03/grupode-trabalho-gt-de-enfrentamento-da-pandemia-sbp-covid-19.

25. Paiva RA, Randow R, Diniz LP, Guerra VA. O Papel do Gestor de Serviços de Saúde: revisão de literatura. Rev Méd Minas Gerais [Internet]. Rev Méd Minas Gerais; 2018. [citadoacesso em 2020 jun Jun 30]; 28(5). Disponível em: http://www.dx.doi. org/10.5935/2238-3182.20180135.

26. Cunha S, Siman A, Brito M. A comunicação como recurso para tomada de decisão de gestores da unidade de atendimento imediato. Braz J Hea Rev [Internet]. Braz J Hea Rev; 2020. [acesso citado em 2020 jul Jul 26]; 3(1): 2374-2383. Disponível em: https://doi.org/10.34119/bjhrv3n2-087.

27. Sousa KO, Barros LM. Estresse e Estratégias de Enfrentamento de Gestores de Saúde [Internet]. Estud Pesqui Psicol [Internet].; 2018. [acesso citado em 2020 jul Jul 26]; 18(2): 496-515. Disponível em: https://doi.org/10.12957/epp.2018.38809.

28. Ximenes MAM, Fontenele NAO, Bastos IB, Macêdo TS, Galindo NNM, Caetano JA et al. Construction and validation of educational booklet content for fall prevention in hospitals [Internet]. Acta Paul Enferm [Internet].; 2019. [acesso citado em 2020 jul Jul 26]; 32(4): 433-441. Disponível em: https://doi.org/10.1590/1982-0194201900059.

29. Gonçalves VM, Pacífico CF, Teixeira PG, Nagami V, Zazula R, Gon MCC. Análise dos materiais educativos sobre diabetes para crianças. [Internet]. 2014. [acesso citado em 2020 jul Jul 26]; 18(1). Disponível em: http://www.seer.ufu.br/index.php/ perspectivasempsicologia/article/view/28576.

30. Souza G, Ribeiro M. Construção de manual sobre cirurgia segura para profissionais de saúde [Internet]. Cogitare Enferm [Internet].; 2017. [acesso citado em 2020 jul Jul 26]; 22(1): 01-05. Disponível em: https://doi.org/22. 10.5380/ce.v22i1.46435.

RECEBIDO:01/08/2020

ACEITO:21/10/2020 